\title{
Evidence for a fruit fly hemangioblast and similarities between lymph-gland hematopoiesis in fruit fly and mammal aorta-gonadal-mesonephros mesoderm
}

\author{
Lolitika Mandal, Utpal Banerjee \& Volker Hartenstein
}

The Drosophila melanogaster lymph gland is a hematopoietic $\operatorname{organ}^{1}$ and, together with prospective vascular cells (cardioblasts) and excretory cells (pericardial nephrocytes), arises from the cardiogenic mesoderm. Clonal analysis provided evidence for a hemangioblast ${ }^{2,3}$ that can give rise to two daughter cells: one that differentiates into heart or aorta and another that differentiates into blood. In addition, the GATA factor gene pannier (pnr) and the homeobox gene tinman (tin), which are controlled by the convergence of Decapentaplegic (Dpp), fibroblast growth factor (FGF), Wingless (Wg) and Notch signaling, are required for the development of all cardiogenic mesoderm, including the lymph gland. Here we show that an essential genetic switch that differentiates between the blood or nephrocyte and vascular lineages involves the Notch pathway. Further specification occurs through specific expression of the GATA factor Serpent (Srp) in the lymph-gland primordium. Our findings suggest that there is a close parallel between the molecular mechanisms functioning in the $D$. melanogaster cardiogenic mesoderm and those functioning in the mammalian aorta-gonadal-mesonephros mesoderm ${ }^{4}$.
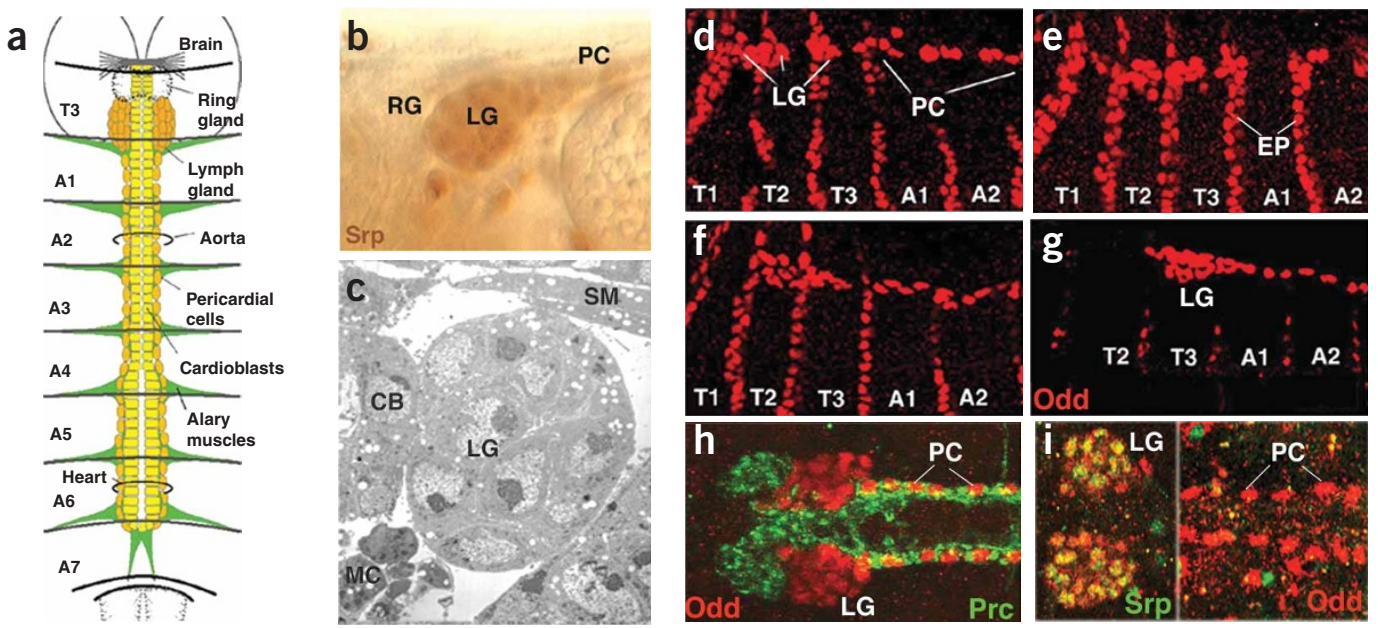

Figure 1 D. melanogaster lymph-gland development. (a) Late embryonic lymph gland and associated structures (dorsal view, anterior is up). (b) Expression of Srp (brown) in late embryonic lymph gland (LG). Visible neighboring organs are the neuroendocrine ring gland (RG) and pericardial nephrocytes (PC). (c) Transmission electron micrograph cross-section of late embryonic lymph gland showing densely packed, prohemocyte cells flanking the aortic cardioblasts (CB). Visible neighboring structures are the somatic muscles (SM) and macrophages (MC). (d-g) Expression of Odd (red) in developing lymph gland (lateral view, dorsal is up) at embryonic stages 13 (d), 14 (e), 15 (f) and 16 (g). In addition to the lymph gland (LG) and pericardial nephrocytes (PC), Odd is expressed in segmental epidermal stripes (EP). The lymph gland develops by fusion of the three Odd-positive clusters (e) and ends up in T3 (g). (h) Expression of Odd (red) in late embryonic lymph gland (LG) and pericardial nephrocytes (PC; dorsal view). Antibody to Prc (green) labels pericardial cells but not lymph gland. (i) Double labeling of late embryonic dorsal vessel with antibodies to Srp (green) and Odd (red). Both signals overlap completely for the lymph gland (LG; left), whereas no Srp staining is seen in pericardial nephrocytes (PC; right).

Department of Molecular Cell and Developmental Biology, University of California Los Angeles, Los Angeles, California 90095, USA. Correspondence should be addressed to V.H. (volkerh@mcdb.ucla.edu) or U.B. (banerjee@mbi.ucla.edu). 


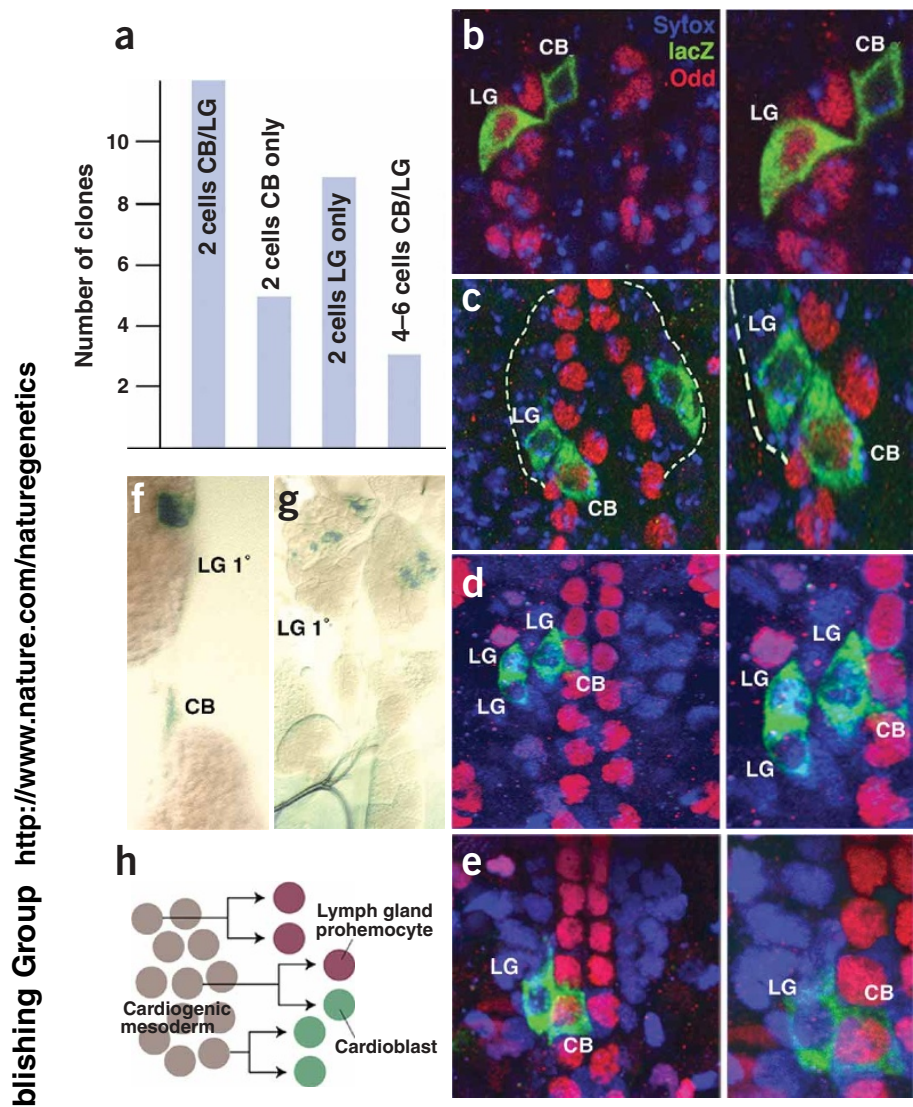

Blood and vascular cells in the vertebrate embryo are thought to derive from oligopotent progenitor cells, called hemangioblasts ${ }^{5}$, that arise in the yolk sac and in the aorta-gonadal-mesonephros (AGM) mesenchyme ${ }^{4}$. A close relationship between blood and vascular progenitors
Figure 2 A hemangioblast-like cell in D. melanogaster. (a) Composition of clones generated by FLP/FRT-induced recombination 3-4 h after fertilization. Of 29 clones located in the aorta and lymph-gland region, 12 were two-cell mixed clones containing a cardioblast and a lymph-gland cell, 5 were pure cardioblast clones, 9 were pure lymph-gland clones and 3 were mixed four-cell clones with cardioblasts and lymph-gland cells. (b) Mixed two-cell clone (green) comprising a lymph-gland cell (Odd-positive, red) and a cardioblast (recognizable by its position, nuclear shape and absence of Odd). (c) Left, mixed two-cell clone; right, pure lymph-gland clone.

Cardioblasts are identified by Mef2 expression (red). (d) Four-cell clone with one cardioblast and three lymph-gland cells doubly labeled with antibodies to Srp (blue) and Mef2 (red). (e) Mixed two-cell clone doubly labeled with antibodies to Srp (blue) and Mef2 (red). Right images in $\mathbf{b}-\mathbf{e}$ are higher magnifications of $\mathbf{b}-\mathbf{e}$, respectively. (f) Clone (blue, X-gal staining) induced in the early embryo and fixed at the late larval stage. The lymph-gland cell has proliferated to produce $\sim 12-16$ cells; the nearby cardioblast has greatly increased in size (owing to polyploidization) but has remained single, as expected. (g) Large lymph-gland clone recovered at larval stage.

(h) Lineage specification of the cardiogenic mesoderm showing that cells can divide exclusively into cardioblast and lymph-gland fates or into a mixture of the two. CB, cardioblasts; LG, lymph gland.

is well established, but in vivo evidence that a single cell can divide to produce a blood cell and an endothelial cell is lacking in vertebrate systems. Similarly, the molecular mechanism that distinguishes between the two lineages is not well understood. To address these issues in a simple, genetically amenable system, we analyzed the genetic control of hematopoiesis in D. melanogaster. Our results show that there are close lineage relationships between hematopoietic and vascular cells, similar to those present in the AGM of mammalian systems. We also provide evidence for conserved cassettes of transcription factors and signaling cascades that limit the pool of hemangioblastic cells and promote the blood versus vascular fate.

In the mature D. melanogaster embryo, the lymph gland is formed by a paired cluster of $\sim 20$ cells flanking the aorta (Fig. 1a). The aorta and heart represent a contractile tube lined by a layer of myoepithelial vascular cells called cardioblasts. The cells flanking the aorta and heart
Figure 3 Developmental origin of the lymph

gland from the cardiogenic mesoderm. (a) Wildtype (WT) lymph gland (anti-Odd labeling, red) at stage 15 (dorsal view). (b-f) Anti-Odd labeling of embryos homozygous with respect to a null allele of tin (b), $d p p(\mathbf{c}), p n r(\mathbf{d}), h t /(\mathbf{e})$ or $w g(\mathbf{f})$. All embryos are stage 15 and in dorsal view. All five genes are required for proper specification of the cardiogenic mesoderm, and in their mutant genetic backgrounds, the lymph gland (LG), cardioblasts (CB) and pericardial nephrocytes $(\mathrm{PC})$ are absent. (g-j) Notch restricts the cardiogenic mesoderm. Prc, which is used to distinguish between lymph-gland and pericardial fate $^{19}$, is present in all pericardial cells (PC) and a subset of cardioblasts (CB), but not in lymph gland (LG). (g) $N^{\text {tsl }}$ homozygous embryo shifted to the restrictive temperature between $6 \mathrm{~h}$ and $8 \mathrm{~h}$ after fertilization (stage 11 ), when Notch signaling restricts the population of cells forming the cardiogenic mesoderm. Inset, wild-type (WT) lymph gland labeled with antibodies to Odd (red) and Prc (green).

$(\mathbf{h}-\mathbf{j})$ Loss of Notch during this phase results in an increase in lymph-gland cells (h) and cardioblasts and pericardial nephrocytes (two rows instead of one of each of these cell types; compare $\mathbf{i}$ and $\mathbf{j}$ ). (k) Wild-type cardioblasts (CB) labeled with antibody to Mef2. (I) Anti-Mef2-labeled $N^{\text {ts } 1}$ embryo shifted between $6 \mathrm{~h}$ and $8 \mathrm{~h}$, showing an increase in the number of cardioblasts (CB).
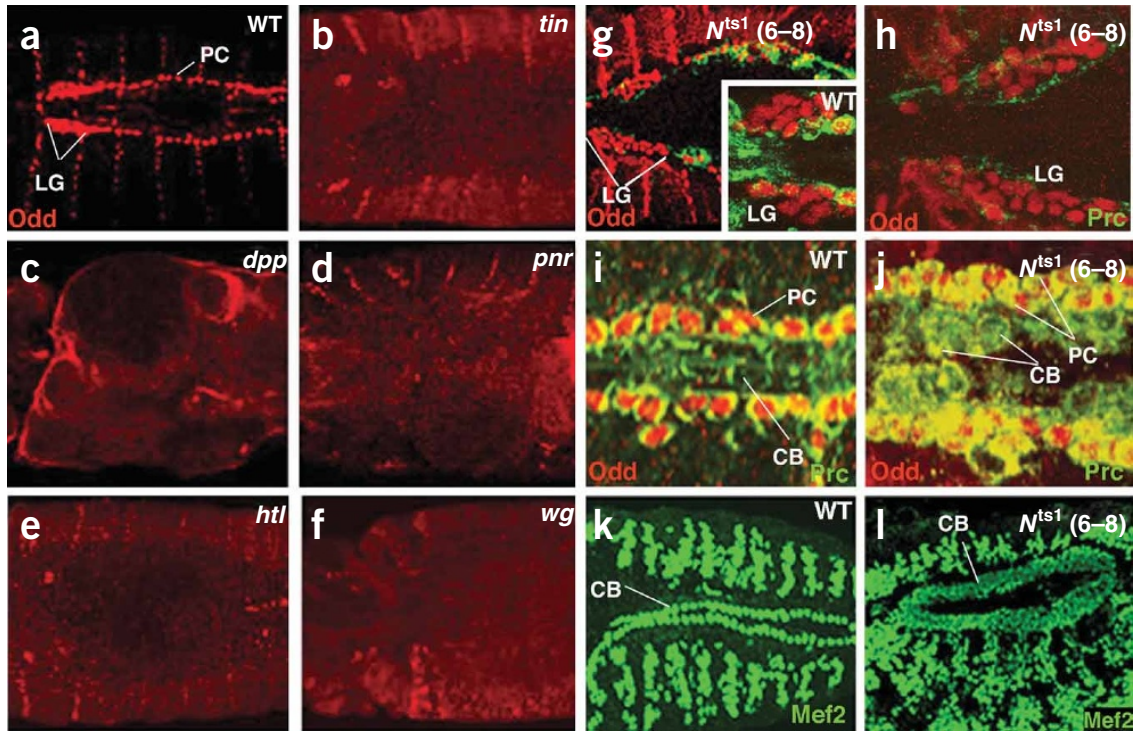
posterior to the lymph gland are the pericardial cells, which function as excretory cells (nephrocytes) ${ }^{6}$. We found that lymph gland progenitors express the prohemocyte marker Srp (Fig. 1b,i) and ultrastructurally resemble prohemocytes that develop at an earlier stage from the head mesoderm (Fig. 1c). Monitoring expression of the zincfinger protein Odd-skipped (Odd) showed that the lymph gland originates from the dorsal thoracic mesoderm (Fig. 1d-h). Odd is expressed in segmental clusters in the dorsal mesoderm of segments T1-A6 (ref. 7). The three thoracic Odd-positive clusters coalesced to form the lymph gland, whereas the abdominal clusters formed the pericardial nephrocytes (Fig. 1g,h).

Lymph-gland progenitors, cardioblasts and pericardial cells are closely related by lineage. We induced labeled 'flipout' (FLP/FRT) clones in embryos aged 3-4 h such that the clones contained only 2-4 cells (Fig. 2a). Of the two-cell clones, $\sim 50 \%$ contained cardioblast and lymph-gland cells (Fig. 2b-e); the other clones comprised either cardioblasts $^{7,8}$ or lymph-gland cells alone (Fig. 2c). We also recovered mixed clones at the late third larval stage (Fig. 2f,g). The finding of mixed clones indicates that the cardiogenic mesoderm of $D$. melanogaster contains oligopotent progenitors that, up to the final division, can give rise both to Srp-positive blood-cell progenitors that form the lymph gland and to vascular cells (Fig. 2h).

The cardiogenic mesoderm forms part of the dorsal mesoderm, 윽 which requires the homeobox protein Tin and the GATA factor Pnr' . In embryos with mutations in tin or pnr, the lymph gland was absent (Fig. 3a,b,d). Maintenance of Tin expression in the dorsal mesoderm ㄱ requires the activity of at least two signaling pathways regulated by Dpp (the D. melanogaster homolog of transforming growth factor- $\beta$ ) and Heartless ( $\mathrm{Htl}$; one of the D. melanogaster homologs of the FGF receptor); the dependence of cardioblast and pericardial nephrocyte development on these signaling pathways is documented ${ }^{10,11}$. We found that lymph-gland progenitors did not develop in loss-offunction $d p p$ and $h t l$ mutants (Fig. 3c,e).

Between $6 \mathrm{~h}$ and $8 \mathrm{~h}$ of development, the dorsal mesoderm splits into the cardiogenic mesoderm and the visceral mesoderm. The cardiogenic mesoderm is regulated positively by Wg and negatively by Notch. Lack of $\mathrm{Wg}$ signaling results in the absence of all cardiogenic lineages ${ }^{12}$ including lymph gland (Fig. 3f). Notch signaling has the opposite effect and restricts cardiogenic mesodermal fate. Notch is active in the dorsal mesoderm from $6 \mathrm{~h}$ to $10 \mathrm{~h}$ of development. We found that eliminating Notch during the first half of this interval by raising embryos homozygous with respect to the temperature-sensitive allele $N^{t s 1}$ at the restrictive temperature resulted in substantially more cardioblasts $^{13}$, pericardial cells and lymph-gland progenitors (Fig. $\mathbf{3 g}-\mathbf{l}$ ).

Lymph-gland progenitors, cardioblasts and pericardial nephrocytes are specified in the cardiogenic mesoderm around the phase of germband retraction $8-10 \mathrm{~h}$ after fertilization. At this stage, we found that Tin, which was initially expressed in the whole cardiogenic mesoderm (Fig. 4a), became restricted to a narrow medial compartment containing the cardioblasts (Fig. 4b). Pnr followed the same restriction (Fig. 4c). Cells located at a more lateral level in the cardiogenic mesoderm gave rise to lymph-gland progenitors (in the thoracic domain) and pericardial nephrocytes (in the abdominal domain) and activated the gene odd (Fig. 4a,b). Slightly later, Srp was expressed in lymph-gland progenitors (Fig. 4d). As reported for the early hemocytes derived from the embryonic head ${ }^{14,15}$, srp is centrally involved in lymph-gland specification. In srp-null embryos, Odd-expressing cells still formed a lymph gland-shaped cluster flanking the aorta, but these cells also expressed the pericardial marker pericardin (Prc) ${ }^{16}$ (Fig. 4e), suggesting that they had lost some aspects of hemocyte precursor identity or gained properties of nephrocytes.
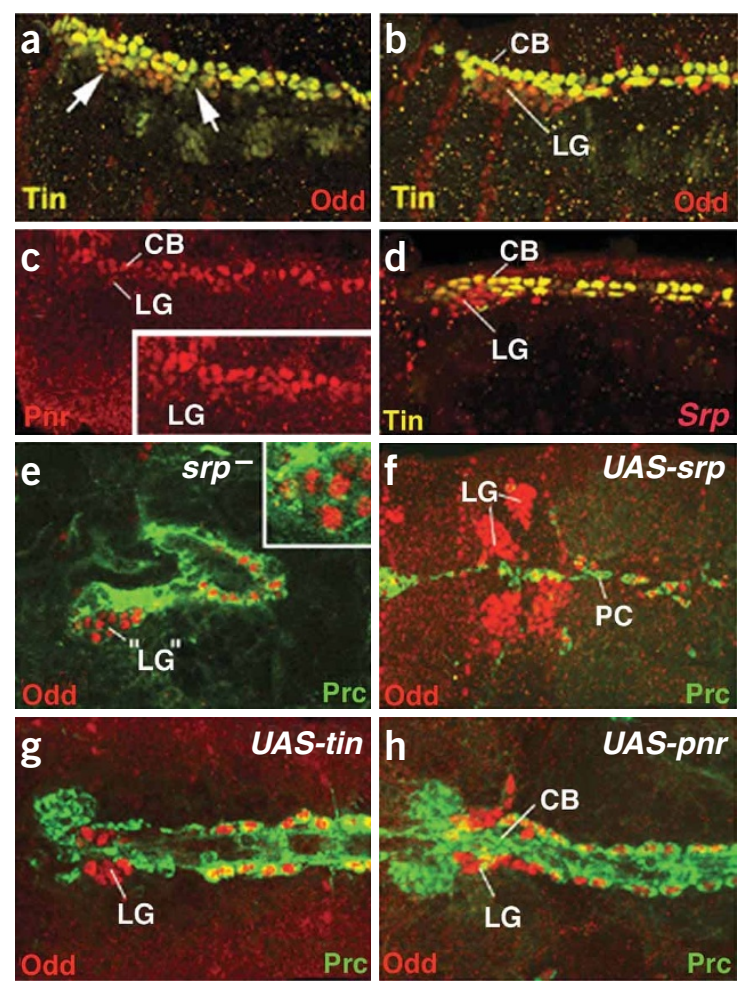

Figure 4 A switch between the transcription factors Tin and Srp is instrumental in lymph-gland specification. (a) Tin (yellow) is expressed in the cardiogenic mesoderm of late-stage-12 embryos. Expression of Tin in the lateral compartment of the cardiogenic mesoderm overlaps with that of Odd (red; Cy3 and fluorescein isothiocyanate-conjugated secondary antibodies to rabbit immunoglobulin were used here because the antibodies to Tin and Odd were both made in rabbit). Arrows indicate the lymph-gland primordium. (b) Expression of Tin is restricted to cardioblasts and a subset of pericardial cells by stage 13. (c) Pnr, like Tin, is restricted to cardioblasts by stage 13 but is still found faintly in lymph-gland and pericardial precursors (inset). (d) Expression of Srp in lymph-gland primordium at stage 13, when Tin withdraws from this lineage. (e) Loss of srp causes transformation of lymphgland primordium to a pericardial cell-like fate, as shown by the expression of Prc. A stage-16 srp mutant embryo in lateral view is shown. Inset, network of Prc immunolabeling surrounding Odd-expressing lymph-gland cells.

(f) Increase in lymph-gland progenitors at the expense of pericardial nephrocytes (PC) in a stage-16 embryo (dorsal view) in which UAS-srp is overexpressed by mef2-Gal4. (g,h) Decrease in lymph-gland progenitors in stage-16 embryos (dorsal view) in which UAS-tin (g) or UAS-pnr (h) is overexpressed by mef2-Gal4. CB, cardioblasts; LG, lymph gland.

As a countercorrelate, ectopic expression of Srp in the whole cardiogenic mesoderm directed by mef2-Gal4 induced pericardial cells to adopt lymph-gland fate (Fig. $4 \mathbf{f}$ ).

Downregulation of tin and pnr in cells in the lateral domain of the cardiogenic mesoderm is essential for lymph-gland specification. Ectopic expression of tin or pnr by twist-Gal4 (or mef2-Gal4) caused a marked reduction in the number of lymph-gland and pericardial cells (Fig. 4g,h). The antagonistic effect of tin on lymph-gland progenitors resembled its earlier role in the head mesoderm that gives rise to the larval blood cells ${ }^{17}$; here too, ectopic expression of $t$ in causes a reduction in the number of hemocytes.

Inhibiting tin and upregulating odd and srp requires input from the Notch signaling pathway. We described a function of Notch at $6-8 \mathrm{~h}$ in specification of the cardiogenic mesoderm (Fig. 3). Reducing Notch 

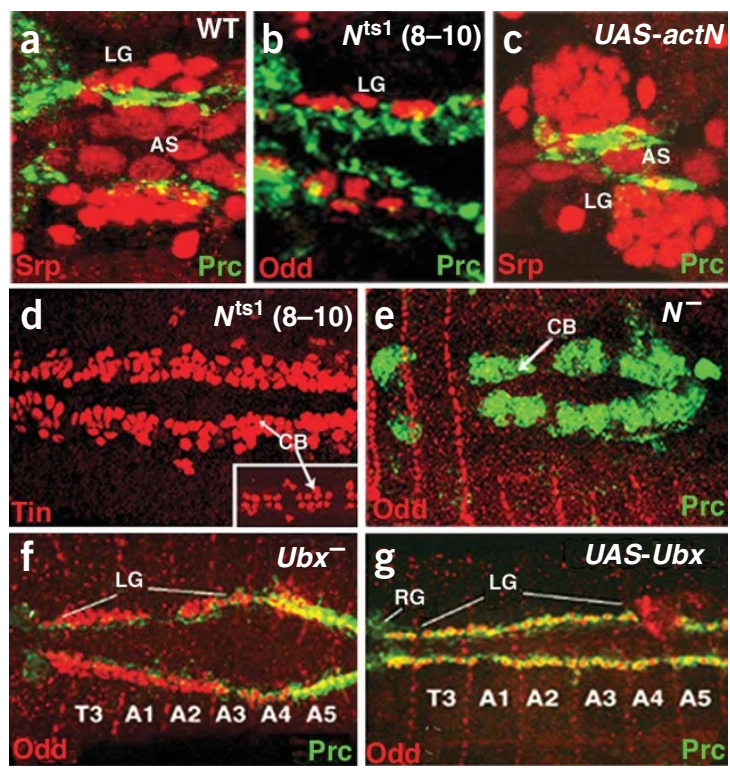

Figure 5 A late phase of Notch activity is required for lymph-gland specification. (a) Late-stage-15 wild-type (WT) lymph gland labeled with antibodies to Srp (red; weak labeling also appears in the amnioserosa, AS). Prc (green) marks the pericardial cells and a subset of cardioblasts.

(b) Reduction of lymph gland (Odd-positive, red; about 6-8 lymph gland cells versus 20 in wild-type) in a stage-15 $N^{\text {ts } 1}$ embryo (dorsal view) reared at the restrictive temperature $8-10 \mathrm{~h}$ after fertilization. (c) Increase in lymph-gland cells (Srp-positive, red; averaging 38 cells versus 20 cells in wild-type) after overexpression of a constitutively active Notch construct by mef2-Gal4. AS, amniosera. (d) Hyperplasia of cardioblasts (labeled by Tin expression, red) in an $N^{\text {ts } 1}$ embryo subjected to the temperature regimen described in $\mathbf{b}$. Inset, wild-type expression of Tin in cardioblasts. (e) Loss of lymph-gland and pericardial cells (Odd-positive, red) and strong hyperplasia of cardioblasts (Prc-positive; green Odd negative) in a stage-16 embryo (dorsal view) carrying the Notch deficiency allele $D f(1) N^{81 \mathrm{k} 1}\left(N^{-}\right)$. (f) Lymph gland is expanded posteriorly into abdominal segments in a stage-15 embryo (dorsal view) lacking the Ubx gene. (g) Lymph-gland progenitors are replaced by pericardial nephrocytes in a stage-15 embryo (dorsal view) in which UAS-Ubx is overexpressed by mef2-Gal4. CB, 50 cardioblasts; LG, lymph gland; RG, ring gland.

function between $8 \mathrm{~h}$ and $10 \mathrm{~h}$ caused an increase in the number of cardioblasts (Fig. 5d) and a concomitant loss of pericardial and lymph-gland cells (Fig. 5a,b). Overexpressing an activated Notch construct caused a marked increase in lymph-gland size (Fig. 5c). This late requirement for Notch signaling was separable from the earlier role of Notch in restricting the overall size of the cardiogenic mesoderm. Thus, the sum total of cardioblasts and pericardial or lymph-gland cells in $N^{\text {ts1 }}$ embryos shifted between $8 \mathrm{~h}$ and $10 \mathrm{~h}$ did not differ substantially from that in wild type, whereas a combined effect on cell number and cell fate was seen in embryos with a Notch deletion (Fig. 5e). In these embryos, the cardiogenic mesoderm was hyperplasic and developed as cardioblasts at the expense of lymphgland progenitors and pericardial nephrocytes. The dual role of Notch in restricting the numbers of a pluripotent progenitor pool and in distinguishing between the progeny of these progenitors is reminiscent of the function of Notch in sense-organ development ${ }^{18}$.

Lymph-gland formation is restricted to the thoracic region by positional cues that are provided by expression of the homeobox proteins of the Antennapedia and Bithorax complex. Specifically, Ultrabithorax (Ubx), which is expressed in segments A2-A5 of the cardiogenic mesoderm ${ }^{19}$, inhibits lymph-gland formation. We found
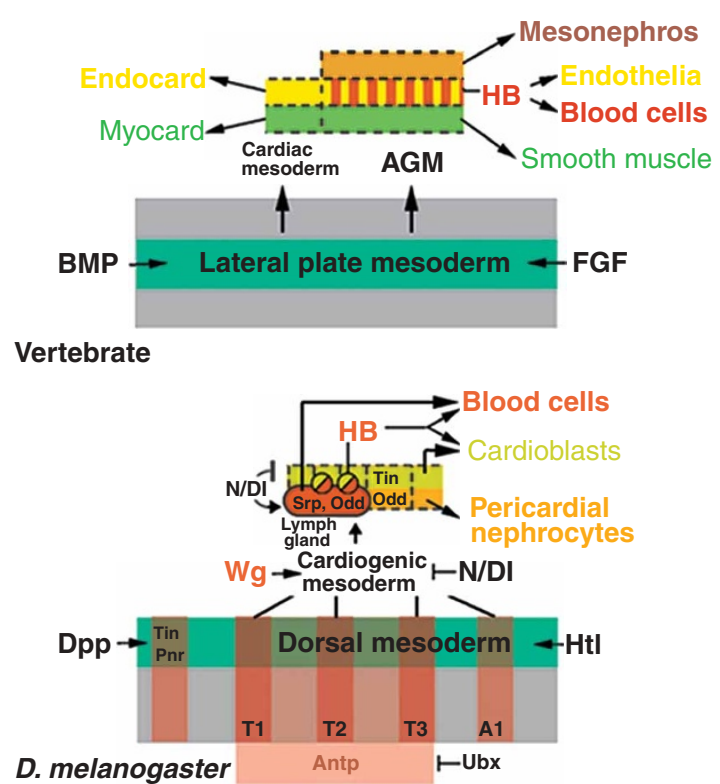

Figure 6 Models of the development of blood progenitors from the mammalian AGM and the D. melanogaster cardiogenic mesoderm. The cardiogenic mesoderm in D. melanogaster (bottom) evolves from the dorsal mesoderm and requires input from the $\mathrm{Htl}$, Dpp, Wg and Notch (N) signaling pathways. The cardiogenic mesoderm then differentiates into lymph gland, vascular cells (cardioblasts) and excretory cells (pericardial nephrocytes). A subpopulation of cardioblasts and lymph-gland cells is derived from one progenitor (hemangioblast; HB). Essential for the differentiation of the cardiogenic mesoderm is the Notch-Delta (DI)-dependent restriction of Tin and Pnr to cardioblasts and the expression of Srp in the lymph gland. In vertebrates (top), similar cell types are derived from a mesodermal domain called the AGM, which also requires the input of FGF, BMP and Wnt signaling. A subset of AGM-derived cells has been proposed to constitute hemangioblasts, which produce blood progenitors and endothelial cells. Antp, Antennapedia.

that loss of Ubx resulted in the expansion of the lymph-gland fate into the abdominal segments (Fig. 5f). Conversely, overexpression of Ubx driven by mef2-Gal4 caused the transformation of lymph-gland progenitors into pericardial nephrocytes (Fig. 5g).

Our findings are suggestive of a model of lymph-gland development in D. melanogaster that is similar to mammalian hematopoiesis (Fig. 6). Lymph-gland progenitors develop as part of the cardiogenic mesoderm that also gives rise to the vascular cells (aorta and heart) and to excretory cells. Similarly, progenitor cells of the blood, aorta and excretory system are closely related both molecularly and developmentally in mammals, where they form part of the AGM. Specification of the cardiogenic mesoderm requires the input of $\mathrm{FGF}^{10}$, $\mathrm{Dpp}^{11}$ and $\mathrm{Wg}^{12}$ signaling, as in vertebrate hematopoiesis, where the AGM region is induced in response to several converging signaling pathways including $\mathrm{FGF}^{20}, \mathrm{BMP}^{21}$ and $\mathrm{Wnt}^{22}$.

Our findings show that in D. melanogaster, the cardiovascular and blood-cell lineages are differentiated by an antagonistic relationship between Tin or Pnr expression in the cardioblasts and Srp expression in the lymph-gland progenitors. In vertebrates, GATA factors also have a pivotal role in specifying different lineages among blood-cell progenitors $^{23}$, although not much is known about what differentiates between blood progenitors as a group and endothelial progenitors. Our results indicate that this step is driven by input from the Notch signaling pathway. In the thoracic cardiogenic mesoderm, Notch antagonizes tin and pnr expression and aortic cardioblast formation, 
and promotes srp expression and the development of lymph-gland progenitors. In vertebrates, Notch signaling is also involved in both blood and vascular development ${ }^{24-26}$. The role of Notch during AGM morphogenesis remains to be investigated.

Cardioblasts and lymph-gland cells can arise from the division of a single cardiogenic mesodermal cell, which should be called a hemangioblast. A previous study induced clones in the cardiogenic mesoderm but used only Tin as a marker ${ }^{8}$. This study also yielded mixed two-cell clones comprising a cardioblast and a nonlabeled cell, which, in light of our findings, must be interpreted as a lymph-gland cell. Hemangioblasts have been proposed in vertebrates, although the definitive experiment in which a precursor is marked and its lineage is tracked has not been done. Blast colony-forming cells that give rise to both lineages in vitro ${ }^{3,6}$ and common markers that belong to both cell types in vivo ${ }^{27}$ have been identified, but direct evidence for the existence of a common precursor has not yet been found. Our study, using genetic analysis of two-cell clones, establishes the existence of such a population in D. melanogaster. On the basis of these results, and given the conservation of the signaling and transcriptional components described here, our prediction is that many cells of the AGM in vertebrates may give rise to only blood or only vascular cells, but a number of intermixed hemangioblasts may give rise to mixed lineages. Future genetic screens aimed at finding components in early lymph윽 gland development will probably identify additional pathways and strategies important for vertebrate hematopoiesis.

\section{METHODS}

Fly strains and antibodies. We used the following D. melanogaster lines and antibodies $(\alpha)$ : $\operatorname{tin}^{346}$, UAS-tin and $\alpha$-Tin (gifts from M. Frasch, Mount Sinai School of Medicine, New York, New York, USA), Act5C-FRT-Draf-FRT-taulac $Z$ and $\alpha$-Odd (gifts from J. Skeath, Washington University School of Medicine, St. Louis, Missouri, USA), $s r p^{6 \mathrm{G}}$ (a gift from R. Reuter, University Tuebingen, Germany), UAS-srp and $\alpha$-Srp (gifts from D. Hoshizaki, University of Nevada, Las Vegas, USA), $h t t^{\mathrm{AB} 42}$ (a gift from A.M. Michelson, Harvard Medical School, Boston, Massachusetts, USA), $p n^{\mathrm{vx} 6}$ and $\alpha$-Pnr (gifts from G. Morata, Universidad Autonoma de Madrid, Spain), UAS-pnr (a gift from M. Haenlin, CNRS/UPS, France), mef2-Gal4 (a gift from E. Chen, University of Texas Southwestern Medical Center, Dallas, Texas), $N^{\text {tsl }}$ (a gift from R. Cagan, hington University School of Medicine, St. Louis, Missouri, USA), $\alpha$-Odd (a gift from D. Kosman, University of California, San Diego), $\alpha$-Prc (a gift from D. Gratecos, Centre Univ. Marseille, France), $\alpha$-Dmef2 (a gift from H.T. Nguyen, Albert Einstein College of Medicine, New York, New York, USA) and $\beta$-galactosidase (Promega). The other fly stocks $\left(d p p^{\mathrm{H} 46}, D f(1) N^{81 \mathrm{~K}}, w g \mathrm{CX} 4, U A S\right.$ $N^{\text {intra }}, U A S-U b x$ and $U b x^{9.22} S b^{\text {sbd }}$ ) were from the Bloomington Stock Center. We carried out histological studies and electron microscopy as described ${ }^{28}$.

Lineage-tracing experiments. We created random lacZ-expressing clones in the cardiogenic region by using the FLP/FRT lineage-tracing system ${ }^{7}$ with some modifications. We heat-shocked $h s$-flp Act5C-FRT-Draf-FRT-tau-lacZ embryos, aged 3-4 h, for $30 \mathrm{~min}$ at $37{ }^{\circ} \mathrm{C}$ to induce Flp recombinase. We then shifted them back to $18{ }^{\circ} \mathrm{C}$ and aged them until stages $15-16$. We fixed and immunostained the embryos by standard methods.

Heat-pulse experiments. At appropriate stages, as indicated in the text, we collected $N^{\text {tsl }}$ embryos at $18{ }^{\circ} \mathrm{C}$, reared them for $2 \mathrm{~h}$ at the restrictive temperature $\left(31{ }^{\circ} \mathrm{C}\right)$ and then transferred them to the permissive temperature $\left(18^{\circ} \mathrm{C}\right.$ ) until stages $15-16$. We used wild-type embryos (Oregon R) subjected to the same temperature regimens as controls.

\section{ACKNOWLEDGMENTS}

We thank R. Cagan, J.A. Campos-Ortega, M. Cho, M. Frasch, D. Gratecos, M. Haenlin, D. Hoshizaki, K. Matthews, A.M. Michelson, G. Morata, H.T. Nguyen, R. Reuter, D. Kosman and J. Skeath for fly stocks and antibodies; and members of the laboratories of V.H. and U.B., particularly S.-H. Jung, for help and advice on the manuscript.

\section{COMPETING INTERESTS STATEMENT}

The authors declare that they have no competing financial interests.

Received 19 February; accepted 29 June 2004

Published online at http://www.nature.com/naturegenetics/

1. Evans, C.J., Hartenstein, V. \& Banerjee, U. Thicker than blood: conserved mechanisms in Drosophila and vertebrate hematopoiesis. Dev. Cell 5, 673-690 (2003).

2. Murray, P.D.F. The development in vitro of the blood of the early chick embryo. Proc. $R$. Soc. Lond. B 11, 497-521 (1932).

3. Choi, K., Kennedy, M., Kazarov, A., Papadimitriou, J.C. \& Keller, G. A common precursor for hematopoietic and endothelial cells. Development 125, 725-732 (1998).

4. Medvinsky, A. \& Dzierzak, E. Definitive hematopoiesis is autonomously initiated by the AGM region. Cell 86, 897-906 (1996).

5. Ema, M. et al. Combinatorial effects of the Flk1 and Tal1 on the vascular and hematopoietic development in the mouse. Genes Dev. 17, 380-393 (2003).

6. Crossley, A.C. The ultrastructure and function of pericardial cells and other nephrocytes in an insect: Calliphora erythrocephala. Tissue Cell 4, 529-560 (1972).

7. Ward, E.J. \& Skeath, J.B. Characterization of a novel subset of cardiac cells and their progenitors in the Drosophila embryo. Development 127, 4959-4969 (2000).

8. Alvarez, A.D., Shi, W., Wilson, B.A. \& Skeath, J.B. Pannier and PointedP2 act sequentially to regulate Drosophila heart development. Development 130, 30153026 (2003).

9. Klinedinst, S.L. \& Bodmer, R. GATA factor Pannier is required to establish competence for heart progenitor formation. Development 130, 3027-3038 (2003).

10. Beiman, M., Shilo, B.Z. \& Volk, T. Heartless, a Drosophila FGF receptor homolog, is essential for cell migration and establishment of several mesodermal lineages. Genes Dev. 10, 2993-3002 (1996)

11. Frasch, M. Induction of visceral and cardiac mesoderm by ectodermal Dpp in the early Drosophila embryo. Nature 374, 464-467 (1995).

12. Wu, X., Golden, K. \& Bodmer, R. Heart development in Drosophila requires the segment polarity gene wingless. Dev. Biol. 169, 619-628 (1995).

13. Hartenstein, A.Y., Rugendorff, A., Tepass, U. \& Hartenstein, V. The function of the neurogenic genes during epithelial development in the Drosophila embryo. Development 116, 1203-1220 (1992).

14. Rehorn, K.P., Thelen, H., Michelson, A.M. \& Reuter, R. A molecular aspect of hematopoiesis and endoderm development common to vertebrates and Drosophila. Development 122, 4023-4031 (1996).

15. Lebestky, T., Chang, T., Hartenstein, V. \& Banerjee, U. Specification of Drosophila hematopoietic lineage by conserved transcription factors. Science 288, 146-149 (2000).

16. Chartier, A., Zaffran, S., Astier, M., Semeriva, M. \& Gratecos, D. Pericardin, a Drosophila type IV collagen-like protein is involved in the morphogenesis and maintenance of the heart epithelium during dorsal ectoderm closure. Development 129, 3241-3253 (2002).

17. Yin, Z., Xu, X.L. \& Frasch, M. Regulation of the Twist target gene tinman by modular cis-regulatory elements during early mesoderm development. Development 124, 4971-4982 (1997)

18. Hartenstein, V. \& Posakony, J.W. A dual function of the Notch gene in Drosophila sensillum development. Dev. Biol. 142, 13-30 (1990).

19. Lo, P.C., Skeath, J.B., Gajewski, K., Schulz, R.A. \& Frasch, M. Homeotic genes autonomously specify the anteroposterior subdivision of the Drosophila dorsal vessel into aorta and heart. Dev. Biol. 251, 307-319 (2002).

20. Nishikawa, M. et al. Role of the microenvironment of the embryonic aorta-gonadmesonephros region in hematopoiesis. Ann. NY Acad. Sci. 938, 109-116 (2001).

21. Marshall, C.J., Kinnon, C. \& Thrasher, A.J. Polarized expression of bone morphogenetic protein-4 in the human aorta-gonad-mesonephros region. Blood 96, 1591-1593 (2000).

22. Orelio, C. \& Dzierzak, E. Identification of 2 novel genes developmentally regulated in the mouse aorta-gonad-mesonephros region. Blood 101, 2246-2249 (2003).

23. Fossett, N. \& Schulz, R.A. Functional conservation of hematopoietic factors in Drosophila and vertebrates. Differentiation 69, 83-90 (2001).

24. Kumano, K. et al. Notch1 but not Notch2 is essential for generating hematopoietic stem cells from endothelial cells. Immunity 18, 699-711 (2003).

25. Lawson, N.D. et al. Notch signaling is required for arterial-venous differentiation during embryonic vascular development. Development 128, 3675-3678 (2001).

26. Schroeder, T. et al. Recombination signal sequence-binding protein $\mathrm{J}_{\kappa}$ alters mesodermal cell fate decisions by suppressing cardiomyogenesis. Proc. Natl. Acad. Sci. USA 100, 4018-4023 (2003).

27. Fehling, H.J. et al. Tracking mesoderm induction and its specification to the hemangioblast during embryonic stem cell differentiation. Development 130, 4217-4227 (2003).

28. Tepass, U., Fessler, L.I., Aziz, A. \& Hartenstein, V. Embryonic origin of hemocytes and their relationship to cell death in Drosophila. Development 120, 1829-1837 (1994). 